\title{
USO DOS MODELOS DE FILAS NO GERENCIAMENTO DO SERVIÇO DE ATENDIMENTO AO CLIENTE BANCÁRIO: UM PANORAMA DA PRODUÇÃO CIENTIFICA NACIONAL E INTERNACIONAL
}

\author{
André Andrade Longaray ${ }^{\text {a*}}$, Paulo Roberto Munhoz ${ }^{\mathrm{b}}$, Vanessa Duarte de Souza ${ }^{\mathrm{c}}$, \\ Thauane Adamoli Amaral ${ }^{\text {d }}$ \\ aPrograma de Pós-Graduação em Administração - PPGA \\ Universidade Federal do Rio Grande - FURG, Rio Grande-RS, Brasil \\ bInstituto de Ciências Humanas e da Informação - ICHI \\ Universidade Federal do Rio Grande - FURG, Rio Grande-RS, Brasil \\ 'Instituto de Ciências Econômicas, Administrativas e Contábeis - ICEAC \\ Universidade Federal do Rio Grande - FURG, Rio Grande-RS, Brasil \\ ${ }^{\mathrm{d} E s c o l a ~ d e ~ E n g e n h a r i a ~ Q u i ́ m i c a ~ e ~ d e ~ A l i m e n t o s ~-~ E Q A ~}$ \\ Universidade Federal do Rio Grande - FURG, Rio Grande-RS, Brasil
}

Recebido 21/03/2017, aceito 29/11/2017

\begin{abstract}
RESUMO
O Brasil tem experimentado nos últimos anos um cenário de crescimento demográfico e expansão do número de pessoas com capacidade de acesso aos serviços do sistema financeiro. $\mathrm{O}$ setor bancário tem estabelecido estratégias para prestar atendimento rápido e eficiente a seus clientes. Uma dessas ações é a busca por uma administração e otimização do tempo de atendimento ao consumidor nas agências. O objetivo deste artigo é descrever e caracterizar a produção científica sobre teoria das filas em âmbito Nacional e Internacional entre os anos de 2006 a 2015. A metodologia utilizada foi um estudo quantitativo, descritivo, instrumentalizado por meio de pesquisa bibliográfica analisada à luz da bibliometria. Como resultado identificou os autores mais prolíficos e os mais citados, os métodos mais aplicados, as temáticas e segmentos estudados.
\end{abstract}

\section{Palavras-chave: Bancos, Teoria das filas, Bibliometria.}

\begin{abstract}
Brazil has experienced in recent years a scenario of population growth and expansion of the number of people with access to financial system services. The banking sector has established strategies to provide fast and efficient service to its clients. One of these actions is the search for an administration and optimization of the time of customer service in the agencies. The aim of this article is to describe and characterize the scientific production on queuing theory in the National and International spheres between the years 2006 and 2015. The methodology used was a quantitative, descriptive study, instrumented through a bibliographical research analyzed in the light of bibliometrics. As a result, he identified the most prolific and most cited authors, the most applied methods, the themes and segments studied.
\end{abstract}

Keywords: Banks, Queuing theory, Bibliometrics.

*Autor para correspondência. E-mail: andrelongaray@gmail.com

DOI: 10.4322/PODes.2017.012 


\section{Introdução}

O setor bancário desempenha importante serviço à população. As agências bancárias prestam atendimento ao público em geral de no mínimo 5 horas diárias ininterruptas com atendimento obrigatório no período de $10 \mathrm{~h}$ às $15 \mathrm{~h}$, horário de Brasília, exceto em algumas datas previstas em normativas estabelecidas pelo Banco Central do Brasil - BACEN (2015).

No ano de 2011, os caixas internos das agencias realizaram 11\% das transações bancárias. Segundo a Federação Brasileira de Bancos - FEBRABAN (2012), esse percentual continua relevante para o setor bancário. Portanto, com o intuito de controlar o tempo de espera nos caixas físicos, todas as agências devem possuir equipamentos que emitam senhas com o horário de chegada (FEBRABAN, 2009).

De acordo com o BACEN (2015), não há regulamentação sobre o tempo de espera em filas, porém existem leis estaduais e municipais que tratam do assunto. A FEBRABAN (2009) estabelece no art. 10 do Sistema de Autorregulação Bancária que nas praças que não possuam regulamentação por lei estadual e municipal, o tempo máximo de espera para atendimento nos guichês de caixa será de até 20 minutos em dias normais e de até 30 minutos em dias de pico. São considerados dias de pico a véspera ou dia útil pós-feriado, o último dia útil do mês e do $1^{\circ}$ ao $10^{\circ}$ dia útil de cada mês. O tempo é contado com anotação do horário e da data da entrada do consumidor na fila até o início efetivo do atendimento pelo caixa.

A geração de filas é uma consequência da incompatibilidade entre a chegada do cliente/usuário e a capacidade de atendimento (Abensur, 2011). As filas podem tornar-se desagradáveis, causando desconforto e frustração para os clientes. Gianesi e Corrêa (1994) afirmam que as filas e a maneira como elas são gerenciadas são aspectos dos mais sensíveis e importantes na percepção do cliente quanto à qualidade do serviço prestado, o que justifica uma preocupação especial com o seu gerenciamento.

Diante disso, emerge a questão de pesquisa do presente trabalho: É possível mapear a produção científica nacional e internacional sobre os estudos da teoria das filas no setor bancário?

Para tanto, o objetivo deste artigo é a caracterização da produção científica nacional e internacional sobre estudos de teoria das filas no setor bancário, por meio de uma análise bibliométrica de artigos publicados.

$\mathrm{O}$ estudo se justifica à medida que a bibliometria permite delinear o estado da arte de determinada temática da ciência, neste caso específico, o emprego da teoria das filas no segmento bancário.

As bases de dados selecionadas para realizar o levantamento da produção científica foram a Web of Science, Science Direct, Portal de Periódicos CAPES e Google Acadêmico. Os artigos disponíveis nessas bases passaram por uma seleção a fim de identificar quais eram relevantes para a pesquisa. Logo, foram utilizadas informações contidas no resumo, palavras-chave e referências com o intuito de identificar autores e instituições prolíferas, autores mais citados, ano de publicação da pesquisa e temas abordados.

Este artigo divide-se em 5 seções. Estabelecido o marco introdutório, a seção 2 expõe o referencial teórico sobre teoria das filas. Na seção 3 é abordado o enquadramento metodológico da pesquisa. A seção 4 evidencia o levantamento bibliométrico realizado no estudo. Por fim, a seção 5 descreve as considerações finais, bem como as limitações encontradas no presente trabalho e sugestões para futuras pesquisas.

\section{Referencial Teórico}

Estudos sobre teoria das filas objetivam dimensionar a capacidade de atendimento em demandas cujos números de canais de recepção são menores do que o número de clientes. No setor bancário, essa desproporcionalidade mostra-se ainda mais proeminente. Segundo Araújo e Carneiro (2008), a presença de filas tem se configurado em uma constante no setor de serviços. Extinguir ou, pelo menos, minimizar este fenômeno, pode representar valor percebido e agregado pelo cliente. 
As possíveis causas das filas nos bancos são diversificação dos serviços oferecidos, redução do quadro de colaboradores associado ao aumento de serviços e falta de interesse dos dirigentes bancários. Entende-se que a maioria da população que utiliza os caixas internos são indivíduos que não possuem conta na agência, desta forma, buscam apenas algum serviço oferecido, por esse motivo não há interesse por parte da agência em atendê-los bem. Além do mais, os dirigentes de bancos partem do princípio que a introdução da tecnologia iria substituir o atendimento pessoal e reduzir o quadro de colaboradores antes mesmo de conhecer os resultados (Araújo e Carneiro, 2008). De acordo com Dienstmann et al. (2010), é necessário agir sobre o problema, ou seja, melhorar o fluxo dos clientes dentro da agência, de maneira que clientes com potencial de novos negócios sejam atendidos com o mínimo de espera, e os processos de baixo valor agregado sejam minimizados ou eliminados da área interna da agência limitando-os à área de autoatendimento.

Para otimização do tempo de espera e dimensionamento do sistema, utiliza-se conceitos de teoria das filas através de fórmulas matemáticas ou simulação computacional (Prado, 2004). A utilização da simulação computacional tem como finalidade representar um modelo real e propor mudanças sem interferência no mesmo (Aragão et al., 2010).

A simulação do modelo real deve ser realizada com base nos elementos compostos em uma fila. São eles o tamanho da população, processo de chegada, processo de atendimento, número de atendentes, disciplina da fila, tamanho médio da fila, capacidade de atendimento e tempo médio de espera na fila (Prado, 2004).

Utilizando-se desses conceitos Bandeira e Rocha (2010), realizaram um estudo de caso em que o problema da demora excessiva encontra-se em outros fatores qualitativos que não são o número de clientes ou de caixas.

Em outra pesquisa sobre o assunto Leitner et al. (2011) identificaram por meio da aplicação da teoria das filas, o número de caixas necessários ao atendimento da demanda local para cumprimento da legislação. A implementação da proposta reduziu o número de reclamações e contribuiu com outras agências.

Por fim, Moro (2010) também contribuiu determinando o número mínimo de atendentes e identificou que o tempo de atendimento dos clientes prioritários são menores do que os clientes da fila comum.

Desta forma, busca-se contribuir com os resultados anteriores, realizando uma análise da produção científica nacional e internacional sobre teoria das filas no setor bancário, a fim de auxiliar em futuras pesquisas acerca do tema. Sendo assim, será realizado um levantamento sem definição de tempo em busca de montar um portfólio bibliográfico para auxiliar futuras pesquisas referentes à Teoria das filas no setor bancário.

\section{Metodologia}

O enquadramento metodológico da pesquisa é definido a partir do propósito do projeto, do caráter, do delineamento da pesquisa, das técnicas de coleta e da análise de dados a ser utilizado (Roesch, 1999).

Segundo Roesch (1999), o propósito da pesquisa é obter informações sobre determinada população. Na presente pesquisa, a população é os artigos publicados sobre teoria das filas, cuja amostra é os artigos selecionados nas bases de dados Scielo, Google acadêmico e Web of Science.

Quanto ao caráter, o estudo é quantitativo, pois a pesquisa bibliométrica busca informações transformando em dados discretos para posteriormente realizar análise estatística.

A bibliometria consiste na aplicação de técnicas estatísticas e matemáticas para descrever aspectos da literatura e de outros meios de comunicação (Araújo, 2006).

A respeito do delineamento a pesquisa é considerada descritiva, porque busca contabilizar em que proporção ou frequência certos eventos acontecem. Com relação a técnica de coleta de dados, a pesquisa adequada é a bibliográfica, pois é através da busca em artigos que são coletadas as informações. No que tange à análise dos dados, foi predominantemente quantitativa, fazendo uso da técnica de bibliometria. 


\section{Análise Bibliométrica}

A análise bibliométrica foi realizada com a análise nas bases de dados Web of Science, Science Direct, Portal de Periódicos CAPES e Google acadêmico, onde buscou-se artigos relacionados a Teoria das filas aplicada ao setor bancário, não se estipulou período de tempo como filtro. As buscas se deram no período de setembro de 2017.

A presente seção explana sobre as etapas da análise bibliométrica. A primeira etapa está relacionada com a coleta de dados, por meio da pesquisa bibliográfica. E a segunda apresenta a análise de dados utilizando a bibliometria (Longaray et al., 2015).

\subsubsection{Coleta de Dados}

A coleta de dados deu-se após a definição das palavras-chave e as bases de dados para iniciar a busca dos artigos. As bases selecionadas para a análise foram a Web of Science, onde utilizou-se o software EndNote para realizar a busca, a Science Direct, Portal de Periódicos CAPES e Google Acadêmico. As palavras-chave utilizadas foram inseridas em duplas, as quais foram Queuing theory and bank e Queuing model and banking service.

$\mathrm{Na}$ base de dados Web of Science foram encontrados 96 artigos, onde encontrou-se 41 artigos a partir das palavras Queuing theory and bank e 55 artigos a partir das palavras Queuing model and banking service. Após a análise de título, palavras-chave e resumo resultaram 14 artigos alinhados à pesquisa.

Na base Science Direct foram encontrados 5987 artigos, dentre os quais 2631 artigos foram a partir das palavras-chave Queuing theory and bank e 3356 artigos a partir das palavras Queuing model and banking service. Após análise de título, palavras-chave e resumo resultaram 20 artigos, realizou-se a leitura dos artigos totalizando 6 artigos alinhados à pesquisa.

No Portal de Periódicos CAPES foram encontrados 3661 artigos, onde 2489 artigos referente as buscas com as palavras-chave Queuing theory and bank e 1172 artigos referentes as palavras-chave Queuing model and banking service. Após a análise de título, palavras-chave e resumo resultaram 444 artigos onde realizou-se a leitura dos artigos resultaram 6 artigos alinhados à pesquisa.

$\mathrm{Na}$ base Google Acadêmico foram encontrados 3674 artigos, onde 3640 artigos foram encontrados a partir das palavras Queuing theory and bank e 34 artigos referentes as palavras Queuing model and banking service. Após a análise de título, palavras-chave e resumo resultaram 24 artigos onde após a leitura dos artigos resultaram em 21.

Por fim, realizou-se a soma dos artigos resultantes em cada base, onde obteve-se 48 artigos, após exclusão dos artigos repetidos obteve-se 33 artigos no portfólio para a realização da bibliometria, os artigos estão localizados no Apêndice A.

$\mathrm{Na}$ seguinte seção serão apresentados os resultados das análises dos artigos que compõem o portfólio.

\subsubsection{Resultados}

Fez-se o estudo das instituições de origem das 33 publicações, entretanto, todas apresentaram o mesmo número de artigo por universidade. No que tange a origem das publicações constituintes do portfólio, 25 artigos foram publicados em revistas, tendo 75,75\% de representatividade, 5 artigos publicados em congressos, representando $15,15 \%$ e 3 publicações de teses, representando $9,1 \%$ do portfólio.

Ao realizar o estudo das revistas onde os artigos foram publicados não houve nenhuma que se destacou, onde todas as revistas apresentaram 1 artigo por revista. $\mathrm{Na}$ análise das teses publicadas, nenhuma universidade se destacou, todas apresentaram mesma representatividade de 1 artigo por instituição. E por fim, ao contabilizar os artigos publicados em congressos, onde todos os congressos apresentaram a mesma representatividade de 1 artigo por evento.

A presente seção será dividida na apresentação dos resultados da análise de ano de publicação e país, autores e citações, palavras-chave e, por fim, a análise das referencias. 


\subsubsection{Ano e País de Publicação}

Ao contabilizar as publicações, pode-se perceber que no ano de 2011 e 2014 foram os anos com o maior número de artigos, com representando 15,15\% cada ano. Na Figura 1, está representado o número de artigos por seu respectivo ano.

Figura 1: Número de publicações por ano.

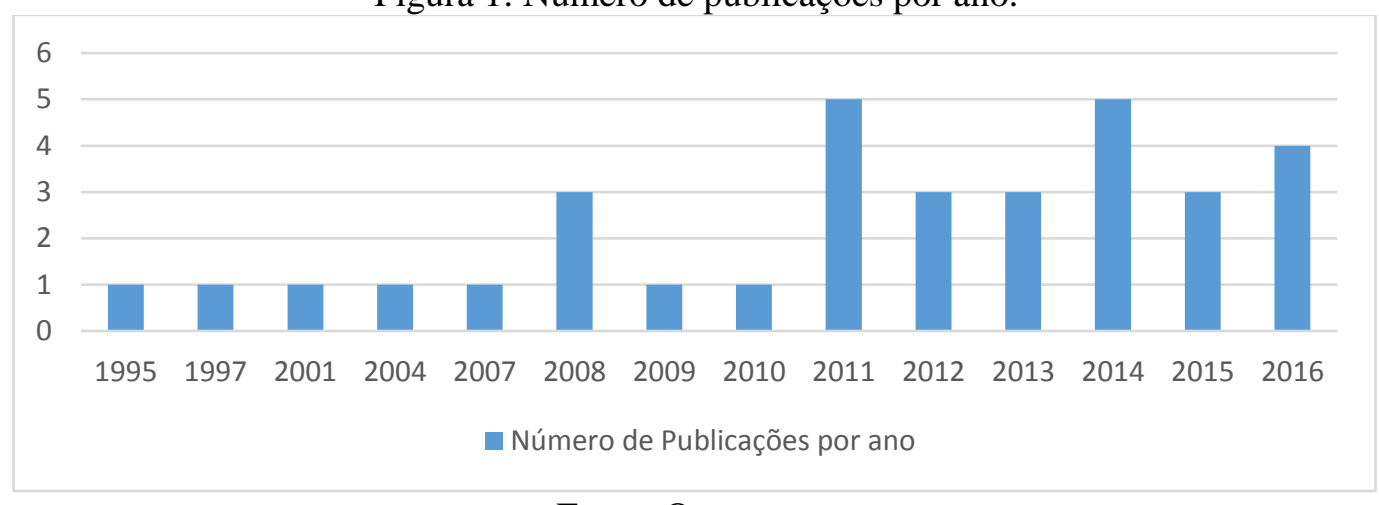

Fonte: Os autores.

Ao analisar o número de publicações por país, obteve-se a China como país que mais produziu sobre o tema da Teoria das filas aplicadas ao setor bancário. Na Tabela 1, está demostrado os países e suas representatividades, onde a China lidera com 24,24\%, Índia com $12,12 \%$ como segundo país de maior número de publicações, e por fim o Brasil apresenta $6,06 \%$ das publicações tendo 2 artigos presentes no portfólio.

Tabela 1: Número de publicações por país.

\begin{tabular}{|c|c|c|}
\hline País & $\mathbf{N}^{\mathbf{0}}$ de publicações & Representatividade \\
\hline China & 8 & $24,24 \%$ \\
\hline Índia & 4 & $12,12 \%$ \\
\hline Nigéria & 3 & $9,1 \%$ \\
\hline Brasil & 2 & $6,06 \%$ \\
\hline Bangladesh & 2 & $6,06 \%$ \\
\hline Canadá & 2 & $6,06 \%$ \\
\hline Espanha & 2 & $6,06 \%$ \\
\hline Gana & 2 & $6,06 \%$ \\
\hline Bahrein & 1 & $3,03 \%$ \\
\hline Israel & 1 & $3,03 \%$ \\
\hline Itália & 1 & $3,03 \%$ \\
\hline Namíbia & 1 & $3,03 \%$ \\
\hline Quênia & 1 & $3,03 \%$ \\
\hline Polônia & 1 & $3,03 \%$ \\
\hline Sri Lanka & 1 & $3,03 \%$ \\
\hline Tailândia & 1 & $3,03 \%$ \\
\hline
\end{tabular}

Fonte: Os autores.

\subsubsection{Autores e Citações}

Ao realizar a analisar os autores dos artigos do portfólio, observou-se que o autor Xiao, H. M. contribuiu com 2 publicações sobre o tema teoria das filas no setor bancário, onde os quais foram publicados em congressos internacionais, o autor Chowdhury, M. S. R. também contabilizou 2 artigos sobre o tema os quais foram publicados em revistas, desta forma, 
apresentaram a maior representatividade de 6,06\% frente aos demais autores que apresentaram 1 artigo (3,03\%) sobre o assunto de teoria das filas aplicado ao setor bancário.

No que tange a análise dos artigos mais citados, obteve-se o artigo "Strategic and queue effects on entry in Spanish banking" sendo citado em 47 publicações. Na Tabela 2, está representado o nome dos artigos presentes no portfólio que foram citados em outras publicações e o número de citações.

Tabela 2: Número de citações.

\begin{tabular}{|c|c|}
\hline Artigo & $N^{0}$ de Citações \\
\hline Strategic and queue effects on entry in Spanish banking & 47 \\
\hline Bayesian control of the number of servers in a GI/M/c queueing system & 26 \\
\hline $\begin{array}{l}\text { Optimizing the Use of Information and Communication Technology (ICT) } \\
\text { in Nigerian Banks }\end{array}$ & 19 \\
\hline $\begin{array}{l}\text { Queuing theory applied to the optimal management of bank excess } \\
\text { reserves }\end{array}$ & 17 \\
\hline Automatic queuing model for banking applications & 13 \\
\hline Improvement of service quality by reducing waiting time for service & 12 \\
\hline $\begin{array}{c}\text { Using queuing theory for evaluating flexibility performance in banking } \\
\text { services }\end{array}$ & 10 \\
\hline $\begin{array}{l}\text { Queuing theory model used to solve the waiting line of a bank-a study on } \\
\text { Islami Bank Bangladesh Limited, Chawkbazar Branch, Chittagong }\end{array}$ & 7 \\
\hline $\begin{array}{c}\text { Reducing lead time in the banking industry: an experimental approach to } \\
\text { the loan granting process }\end{array}$ & 5 \\
\hline $\begin{array}{c}\text { Simulation based alternative strategies in risk and service guarantee } \\
\text { tradeoffs in the international funds transfer system at a large Canadian } \\
\text { Bank }\end{array}$ & 3 \\
\hline $\begin{array}{c}\text { The value of Value-at-Risk: A theoretical approach to the pricing and } \\
\text { performance of risk measurement systems }\end{array}$ & 2 \\
\hline The Optimal Bank Service Based on The Queuing Theory & 2 \\
\hline $\begin{array}{c}\text { An application of OR and IE technology in bank service system } \\
\text { improvement }\end{array}$ & 2 \\
\hline $\begin{array}{c}\text { Automated Queuing And The Experience Of Kenya Commercial Bank } \\
\text { Retail Customers In Nairobi Kenya }\end{array}$ & 1 \\
\hline The queuing theory application in bank service optimization & 1 \\
\hline
\end{tabular}
Fonte: Os autores.

Os demais artigos que constituem o portfólio não foram citados em outras publicações, apresentando 0 citações, desta forma, não foram inseridas na tabela.

\subsubsection{Palavras-chave}

Realizou-se a análise das palavras-chave presentes nas publicações, onde contabilizou-se as palavras-chave contidas nos artigos presentes no portfólio. Teve-se como resultado a palavrachave Queing theory como a mais representativa nas publicações, onde esteve presente em 9 artigos, seguida da palavra Queuing system como a segunda mais presente nas publicações (Tabela 3).

No que tange a comparação das palavras-chaves utilizadas nas buscas nas bases de dados serem Queuing theory and bank e Queing model and banking service, obteve-se apenas a primeira combinação com maior representatividade nas palavras-chave dos artigos que compõem o portfólio. 
Tabela 3: Número de palavras-chave presente nos artigos.

\begin{tabular}{|c|c|c|}
\hline Palavras-chave & $\mathbf{N}^{\mathbf{0}}$ de artigos & Representatividade \\
\hline Queuing theory & 9 & $27,27 \%$ \\
\hline Queuing system & 4 & $12,12 \%$ \\
\hline Bank & 3 & $9,09 \%$ \\
\hline Waiting time & 3 & $9,09 \%$ \\
\hline Queue & 2 & $6,06 \%$ \\
\hline Service rate & 2 & $6,06 \%$ \\
\hline Service & 2 & $6,06 \%$ \\
\hline
\end{tabular}

Fonte: Os autores.

\subsubsection{Referências}

Para a análise das referências foram analisadas as referências presentes nas publicações constituintes do portfólio bibliográfico, onde encontrou-se 428 citações. Na Tabela 4, foram expostos os resultados dos autores mais representativos, onde o autor Ausín, M. C. foi citado em 4 artigos, seguido dos autores Hillier, F. S.; Hoel P. G.; Kleinrock, L.; Parzen, E. e Taha, A. H. os quais foram citados em 3 publicações cada.

Tabela 4: Autores mais citados.

\begin{tabular}{|c|c|}
\hline Autor & $\mathbf{N}^{\circ}$ de Artigos \\
\hline Ausín, M. C. & 4 \\
\hline Hillier, F. S. & 3 \\
\hline Hoel P. G & 3 \\
\hline Kleinrock, L. & 3 \\
\hline Parzen, E. & 3 \\
\hline Taha, A. H. & 3 \\
\hline Armero, C. & 2 \\
\hline Artzner, P. & 2 \\
\hline Bharucha-Reid, A. T. & 2 \\
\hline Chase, R. E. T. & 2 \\
\hline Cooper R. B. & 2 \\
\hline Crouhy, M. & 2 \\
\hline Dhar, S. K. & 2 \\
\hline Dijk, N. M. & 2 \\
\hline Essinger, J. & 2 \\
\hline Feller, W. & 2 \\
\hline Gross, D. & 2 \\
\hline Hogg, R. V. & 2 \\
\hline Howard, R. A. & 2 \\
\hline Hui, L. & 2 \\
\hline Kapoor V.K. & 2 \\
\hline Katz, K. & 2 \\
\hline Kendal, M. G. & 2 \\
\hline Khinchin, A. J. & 2 \\
\hline Lee, A. M. & 2 \\
\hline Levin, R. L. & 2 \\
\hline Lillo, R.E. & 2 \\
\hline Lindgren, B. W. & 2 \\
\hline Little, J. D. C. & 2 \\
\hline Maister, D. H. & 2 \\
\hline Meyer, P.H. & 2 \\
\hline
\end{tabular}




\begin{tabular}{|c|c|}
\hline Montgomery, D.C. & 2 \\
\hline Pei X. & 2 \\
\hline Pidd, M. & 2 \\
\hline Ross, S. & 2 \\
\hline Ross, S. M. & 2 \\
\hline Song, H. & 2 \\
\hline Spiegel, M.R. & 2 \\
\hline Taylor.S. & 2 \\
\hline Tijms H.C. & 2 \\
\hline Wagner, H. M. & 2 \\
\hline Wang X. G. & 2 \\
\hline Winston, W. L. & 2 \\
\hline Wiper, M. P. & 2 \\
\hline Xiao, H. M. & 2 \\
\hline
\end{tabular}

Fonte: Os autores.

\section{Considerações finais}

O presente estudo teve como objetivo a caracterização da produção científica sobre teoria das filas no setor bancário por meio de uma análise bibliométrica de artigos disponíveis nas bases de dados Web of Science, Science Direct, Portal de Periódicos CAPES e Google Acadêmico. Para a coleta de dados foi definida as palavras-chave e inseridas nas bases de dados e após realizou-se a análise dos artigos que se alinhavam ao tema de pesquisa. Quanto ao estudo, buscou-se alinhar todas as pesquisas referentes ao tema, onde estudou as palavras-chave, instituições, revistas, congressos, autores e referências de cada artigo que constituiu o portfólio resultante da bibliometria.

Como resultado, percebeu-se a pouca produção nacional referente à Teoria das filas no setor bancário, entretanto, observou-se que a China apresentou predominância nas publicações referentes ao tema de análise. Não houve a definição de universidade, revistas e congressos que mais produziram, de fato, houve apenas 1 publicação de cada termo acerca da Teoria das filas em bancos.

Quanto aos artigos presentes no portfólio, descrevem um modelo ou algoritmo a fim de auxiliar na diminuição das filas no setor bancário ou demonstram a aplicação da Teoria das filas em bancos.

Entretanto, ao realizar as buscas nas bases de dados percebeu-se um número volumoso de artigos, onde os quais eram sobre a Teoria das filas aplicadas em algum meio, e após a filtragem observou-se um número baixo de publicações aplicados ao setor bancário. Dessa forma, poucos artigos se alinharam ao tema de pesquisa, obtendo poucas publicações nacionais referentes ao tema.

Frente ao exposto, tem-se como limitação a baixa produção referente à Teoria das filas no setor bancário. Desta forma, a partir do estudo realizado, busca-se auxiliar futuras pesquisas sobre a Teoria das filas no setor bancário, a fim de contribuir na produção cientifica sobre o tema de pesquisa.

\section{Referências}

Abensur, E. O. Banking operations using queuing theory and genetic algorithms. Produto \& Produção, v. 12, n. 2, p. 69-86, 2011.

Aragão, A. P., Arica, J., Sales, M. V. S. e Santos, R. B. Simulação do processo produtivo de cerâmica vermelha em Campos dos Goytacazes - RJ. In: Anais do VI Congresso Nacional de Engenharia Mecânica, Campina Grande. ABCM, 2010. 
Araújo, C. A. Bibliometria: evolução histórica e questões atuais. Em Questão, v. 12, n.1, p.1132, 2006.

Araújo, C. A. S. e Carneiro, T. C. J. Filas nos bancos: Por que a tecnologia da informação não resolve? A percepção dos gerentes sobre causas e prováveis solução. Revista Eletrônica de Administração, v. 14, n. 3, p. 569-593, 2008.

BACEN - Banco Central do Brasil. 2015. Atendimento Bancário. Disponível em: http://www.bcb.gov.br/?ATENDIMENTOBANCARIOFAQ. Acesso em: 23/08/2015.

Bandeira, C. R. P. P. e Rocha, S. P. B. Otimização de atendimento bancário: Estudo de caso em uma agência bancária em Aracaju-SE. In: Anais do XXX Encontro Nacional de Engenharia de Produção, São Carlos. ABEPRO, 2010.

Dienstmann, G. H., Azevedo, D. C. e Demartini, F. J. Simulação computacional do processo de atendimento ao público numa agência bancária de forma a maximizar a eficiência e rentabilidade. In: Anais do XXX Encontro Nacional de Engenharia de Produção, São Carlos. ABEPRO, 2010.

FEBRABAN - Federação Brasileira de Bancos. Autorregulação Bancária. 2009. Disponível em: https://portal.febraban.org.br. Acesso em: 20/12/2017.

FEBRABAN - Federação Brasileira de Bancos. Pesquisa CIAB. A Sociedade Conectada: Setor Bancário em Números, Tendências Tecnológicas e Agenda Atual. 2012. Disponível em: http://www.febraban.org.br/7Rof7SWg6qmyvwJcFwF7I0aSDf9jyV/sitefebraban/Pesquisa\%20 CIAB\%20FEBRABAN\%202012.pdf. Acesso em: 20/09/2015.

Gianesi, I. G. N. e Corrêa, H. L. Administração Estratégica de Serviços: Operações para a satisfação do cliente. São Paulo: Atlas, 1994.

Leitner, C. P. S., Sznitowski, A. M. e Geier, L. Dimensionamento do número de caixas executivos em relação à demanda e tempo de atendimento: um estudo de caso em uma agência bancária no interior do estado de Mato Grosso. INGEPRO - Inovação, Gestão e Produção, v. 03, n. 04, p. 24-33, 2011.

Longaray, A. A., Junior, T. L. P., Munhoz, P. R., Geri, F. S. e Castelli, T. M. Caracterização da Produção Científica Brasileira sobre a aplicação de métodos multicritério de apoio à decisão: Uma análise das publicações entre 2004-2013. In: Anais do XXXV Encontro Nacional de Engenharia de Produção, Fortaleza. ABEPRO, 2015.

Moro, D. S. Dimensionamento de canais de atendimento em uma agência bancária. Trabalho de Conclusão de Curso (Graduação em Engenharia de Produção) - Universidade Anhembi Morumbi, São Paulo-SP, 2010.

Prado, D. Teoria das Filas e da Simulação. Belo Horizonte-MG: Editora Desenvolvimento Gerencial, 1999.

Roesch, S. M. A. Projetos de Estágio e de Pesquisa em Administração: Guia para Estágio Trabalhos de Conclusão, Dissertações e Estudos de Caso. 2 ed. São Paulo: Atlas, 1999. 


\section{Apêndice A}

[1] AL-Jumaily, A. S. A. e AL-Jobori, H. K. T. Automatic queuing model for banking applications. (IJACSA) International Journal of Advanced Computer Science and Applications, v. 2, n. 7, p. 11-15, 2011.

[2] Agboola, A. A. e Salawu, R. O. Optimizing the use of information and communication technology (ICT) in Nigerian banks. Journal of Internet Banking and Commerce, v. 13, n. 1, p. $1-15,2008$.

[3] Agyei, W., Asare-Darko, C. e Odilon, F. Modeling and analysis of queuing systems in banks: A case study of Ghana Commercial Bank Ltd. Kumasi Main Branch. International Journal of Scientific \& Technology Research, v. 4, n. 7, p. 160-163, 2015.

[4] Ausín, M. C., Lillo, R. E. e Wiper, M. P. Bayesian control of the number of servers in a GI/M/c queueing system. Journal of Statistical Planning and Inference, v. 137, n. 10, p. 30433057, 2007.

[5] Caridi, M., Cigolini, R., Urciuoli, M. e Villa, A. N. Reducing lead time in the banking industry: an experimental approach to the loan granting process. Production Planning \& Control, v. 19, n. 3, p. 198-211, 2008.

[6] Cherenet, M. Contribution of Queue Management System on customers satisfaction in the case of Commercial Bank of Ethiopia Branches in Addis Ababa. Tese (Doutorado em Administração) - Universidade St. Mary’s, Nova Escócia, Canadá, 2014.

[7] Chowdhury, M. S. R. Queuing theory model used to solve the waiting line of a bank-a study on Islami Bank Bangladesh Limited, Chawkbazar Branch, Chittagong. Asian Journal of Social Sciences \& Humanities, v. 2, n. 3, p. 468-478, 2013.

[8] Chowdhury, M. S. R., Rahman, M. T. e Kabir, M. R. Solving of waiting lines models in the bank using queuing theory model the practice case: Islami Bank Bangladesh Limited, Chawkbazar Branch, Chittagong. IOSR Journal of Business and Management, v. 10, n. 1, p. $22-$ 29, 2013.

[9] de Lima, V. C., Anholon, R., Rampasso, I. S. e Quelhas, O. L. G. Application of queuing theory in banking services. Revista Produção Online, v. 16, n. 1, p. 210-241, 2016.

[10] Deng, Q. e Wei, X. Study of poisson process and the queuing theory in the bank queuing problem. Journal of Hainan Normal University (Natural Science), v. 1, n. 3, 2014.

[11] Filipowicz, B. e Bieda, B. Application of queuing systems with many classes of customers for structural optimisation of banks. IFAC Proceedings Volumes, v. 30, n. 19, p. 149-154, 1997.

[12] Fuentelsaz, L. e Gomez J. Strategic and queue effects on entry in Spanish banking. Journal of Economics \& Management Strategy, v. 10, n. 4, p. 529-563, 2001.

[13] Hettiarachchi, A. T. Application of queuing theory to enhance the operational efficiency of the bank. Tese (Mestrado em Engenharia) - Departamento de Matemática, Universidade de Moratuwa, Sri Lanka, 2016.

[14] Koka, T. A. e Badshah, V. H. Analysis of queuing theory in a bank. International Journal of Applied Research, v. 2, n. 8, p. 731-734, 2016. 
[15] Ling, L., Liang, L., Hu, Z. e Yang, F. Effect of upper and lower withdrawal limits on queue length. Applied Mathematics and Computation, v. 218, n. 7, p. 3834-3846, 2011.

[16] Mohamud, A. A. Automated queuing and the experience of Kenya Commercial Bank retail customers in Nairobi Kenya. Tese (Mestrado em Administração) - Universidade de Nairobi, Quênia, 2016.

[17] Mutingi, M., Mapfaira, H., Moakofi, N. P. K., Moeng, S. A. and Mbohwa, C. Simulation and analysis of a bank queuing system. In: Anais da International Conference on Industrial Engineering and Operations Management, Dubai. IEEE, 2015. p. 1-6.

[18] Ngamsirijit, W. Using queuing theory for evaluating flexibility performance in banking services. International Journal of Services and Operations Management, v. 12, n. 4, p. 387-404, 2012.

[19] Paradi, J. C., Marr, C., Wong, M. e Yan, G. Simulation based alternative strategies in risk and service guarantee tradeoffs in the international funds transfer system at a large Canadian Bank. IEEE Transactions on Engineering Management, v. 42, n. 4, p. 387-396, 1995.

[20] Pei, X. An application of OR and IE technology in bank service system improvement. In: Anais da International Conference on Industrial Engineering and Engineering Management, Cingapura. IEEE, 2008. p. 638-642.

[21] Rohatgi, P. e Shrivastava, N. Queuing analysis of automatic Teller machine (ATM) services in India. International Journal of Agricultural and Statistics Sciences, v. 7, n. 1, p. 167177, 2011.

[22] Sahu, C. e Sahu, S. Implementation of single channel queuing model to enhance banking services. BEST: International Journal of Management, Information Technology and Engineering, v. 2, n. 7, p. 71-78, 2014.

[23] Sarkar, A., Mukhopadhyay, A. R. e Ghosh, S. K. Improvement of service quality by reducing waiting time for service. Simulation Modelling Practice and Theory, v. 19, n. 7, p. 1689-1698, 2011.

[24] Taufemback, C. e da Silva, S. Queuing theory applied to the optimal management of bank excess reserves. Physica A: Statistical Mechanics and its Applications, v. 391, n. 4, p. 13811387, 2012.

[25] Thompson-Nunoofio, E. The application of queuing theory to customer service at selected branches of the Standard Chartered Bank Ghana Limited. Tese (Doutorado). Universidade de Ghana, Ghana, 2004.

[26] Ullah, A., Zhang, X.-D., Iqbal, K. e Ayat, M. Sub-optimization of bank queuing system by qualitative and quantitative analysis. In: Anais da 11th International Conference on Service Systems and Service Management, Beijing, China. IEEE, 2014.

[27] Wen-Jing, C. A. I., e Lian-Sheng, G. E. Optimization of bank counter setting based on queuing theory. Journal of Shandong University (Engineering Science), v. 3, n. 6, 2013.

[28] Wiener, Z. The value of Value-at-Risk: A theoretical approach to the pricing and performance of risk measurement systems. Journal of Economics and Business, v. 64, n. 3, p. 199-213, 2012. 
[29] Williams, H. T., Ogege, S. e Ideji, J. O. An empirical analysis of effective customers service on Nigeria banks profitability. (A queuing and regression approach). Asian Economic and Financial Review, v. 4, n. 7, p. 864-876, 2014.

[30] Xiao, H. e Zhang, G. The queuing theory application in bank service optimization. In: Anais da International Conference on Logistics Systems and Intelligent Management, Harbin, China. IEEE, 2010.

[31] Xiao, H. M.; Zhang, G. Z. The Optimal Bank Service Based on the Queuing Theory. In: Anais da $8^{\text {th }}$ International Conference on Information and Management Sciences, Kunming \& Banna, China, 2009.

[32] Yusuf, M. O., Blessing, N. e Kazeem, A. O. Queuing theory and customer satisfaction: A review of performance, trends and application in banking practice (A study of First Bank Plc Gwagwalada, Abuja Branch). European Journal of Business and Management, v. 7, n. 35, p. 90-96, 2015.

[33] Zheng, H. Analysis and restoration strategies for waiting for bank services. Contemporary Logistics, n. 4, p. 83-86, 2011. 